\title{
Especificaciones metodológicas para el desempeño docente en nivelación de carreras de educación en el marco del modelo pedagógico universitario
}

\section{Methodological specifications for teacher performance in leveling education careers within the framework of the university pedagogical model}

\author{
Pérez Chávez Luis Fernando \\ Luis Alberto Mera Cabezas \\ Universidad Nacional de Chimborazo, Ecuador
}

Autor para correspondencia: lperez@unach.edu.ec, lmera@unach.edu.ec Fecha de recepción: 15 de diciembre 2017 - Fecha de aceptación: 26 de febrero de 2018

Resumen: El objetivo del trabajo es identificar las metodologías aplicadas por docentes en nivelación de la Facultad de Ciencias de la Educación, Humanas y Tecnologías de la Universidad Nacional de Chimborazo, también la apreciación que de esas metodologías tienen los estudiantes. Se elaboró un cuestionario con interrogantes del currículo oficial; instrumento validado por expertos y aplicado a docentes y muestra de estudiantes. El hallazgo es que hay correlación entre las apreciaciones de los dos grupos de actores consultados; sin embargo, el criterio de estudiantes es de menor frecuencia de utilización de esas actividades. Como consecuencia se estructura una propuesta de especificaciones metodológicas guiadas por el modelo pedagógico institucional; estrategia que luego de la validación entrará en pilotaje con una muestra de docentes y estudiantes. La conclusión es que esta metodología debe cursar el ciclo de la mejora continua como teoría científica que guie la cultura del trabajo académico; además que las metodologías hay que innovar constantemente.

Palabras claves: metodología; educación superior; modelo pedagógico

\begin{abstract}
The objective of this research is to identify the methodologies applied by the teachers of leveling in Education Faculty, Humanities and Technology Sciences of the National University of Chimborazo, as well as the appreciation according these methodologies by the students. A questionnaire was elaborated with questions of the official curriculum; this is an instrument validated by experts and this was applied to teachers and also a sample to students. The finding shows that there is a correlation between the opinions of the two stakeholder groups consulted; however, the student criteria is less frequently used for these activities. As a consequence, a proposal of methodological specifications guided by the institutional pedagogical model is structured; this strategy after validation will enter piloting with a sample to teachers and students. The conclusion is that this methodology must follow the cycle of continuous improvement as a scientific theory, this guides the culture of academic research; in addition, methodologies must be constantly innovated.
\end{abstract}

Key words: methodology; higher education; pedagogical model 


\section{Introducción}

\section{Problema}

Es pertinente presentar el problema como la gran interrogante: ¿Hay relación entre la apreciación de las actividades metodológicas utilizadas por los docentes para facilitar los aprendizajes y lo referido por los estudiantes? En una descripción preliminar de esta gran interrogante, se conoce que, uno de los elementos centrales del currículo es la metodología, allí se opera lo planeado mediante una serie de actividades organizadas secuencialmente y coherentes para el o los objetivos definidos; son partícipes directos de esa metodología los docentes y estudiantes, en consecuencia cada uno percibe la presencia o ausencia de actividades y valora la significancia para fines del aprendizaje.

\section{Importancia del problema}

Las actividades que al ser desarrolladas, dan forma a la metodología, si no se ejecutan en un marco de motivación, valoración de su necesidad, uso de recursos, empatía y más elementos condicionantes, no son de impacto y no lograrán el efecto buscado.

La modernidad actual implica otras perspectivas y acciones de docentes y estudiantes, lo que hace pocos años fue de importancia, puede no serlo hoy. Se requiere nuevas competencias de docentes por el avance vertiginoso de la ciencia y tecnología, más por la exigencia de las nuevas generaciones; implica aprender a desaprender, esto es dejar fuera del quehacer docente las actividades que ya no son pertinentes a esta época, por ejemplo el dictado de los contenidos para que el estudiante en una fecha específica memorice y entregue la información en una evaluación; hay que repensar en las actividades docentes desde la planificación, ejecución y evaluación del currículo en sus niveles macro, meso y micro, en ese contexto considerar el quehacer del estudiante desde sus intereses hasta lo que la sociedad y el campo laboral requiere en el caso de estudiantes de carreas de tercer nivel.

En lo referido a esta investigación, se entiende que, "Este cambio de planteamiento impone un cambio en la metodología a seguir en cuanto al desarrollo de la labor docente" (Jenaro, 2012). Si no está presente hay que desarrollar las competencias de planificar y hacer uso de estrategias para facilitar los aprendizajes, eso no se logra solo con revisiones teóricas y tampoco con prácticas intuitivas y bien intencionadas.

Esto implica la revisión integral de las metodologías que utilizan los docentes, acción que sugiere análisis de las actuaciones individuales y de las relaciones interpersonales en el desempeño. En general revisar y mejorar los procesos de aprendizaje porque la metodología por sí sola no garantiza el logro de objetivos propuestos, se engrana con los demás elementos del currículo para tener resultados valederos.

Como marco normativo relacionado con el problema investigado, según el Artículo 81 de la Ley Orgánica de Educación Superior, en Ecuador, "el ingreso a las instituciones de educación superior públicas estará regulado a través del Sistema de Nivelación y Admisión..." (Asamblea Nacional, 2010). 
El Sistema de Nivelación y Admisión (SNNA), trabaja en dos grandes responsabilidades, una para la inscripción, evaluación y asignación de cupos y otra con la estructura y vigilancia de ejecución de currículo para desarrollar competencias en bachilleres como base para la formación en carreras de tercer nivel.

El SNNA es una institución del Estado que a nivel nacional tiene como misión: "Diseñar e implementar políticas públicas que regulan el acceso a la Educación Superior Pública" (SNNA, 2017).

Producto de esas políticas nacionales, en el caso particular de la Universidad Nacional de Chimborazo (UNACH) y Facultad de Ciencias de la Educación, Humanas y Tecnologías (FCEHT), el acceso es reducido, a tal punto que, varias carreras no abren sus primeros niveles. La tabla 1 permite observar esa realidad.

Tabla 1. Estudiantes matriculados y condicionados en carreras de la FCEHT

\begin{tabular}{llcc}
\hline Para licenciatura & $\begin{array}{l}\text { Matriculados } \\
\text { nivelación }\end{array}$ & $\begin{array}{c}\text { Con } \\
\text { puntaje }\end{array}$ & $\begin{array}{l}\text { Condicionados } \\
\text { educación }\end{array}$ \\
\hline Cultura física & 27 & 2 & 25 \\
Biología química y laboratorio & 26 & 6 & 20 \\
Ciencias exactas & 32 & 1 & 31 \\
Ciencias sociales & 23 & & 29 \\
Diseño grafico & 36 & & 22 \\
Educación básica & 32 & 3 & 26 \\
Educación parvularia e inicial & 26 & 4 & 36 \\
Idiomas ingles & 28 & 2 & 189 \\
Informática aplicada a la educación & 37 & 1 & \\
Psicología educativa orientación vocacional y & & & \\
familiar & 27 & & \\
Total 10 carreras & 294 & 19 & \\
\hline
\end{tabular}

Fuente: (Coordinación SNNA UNACH, 2016)

La tabla muestra que, hay 189 estudiantes condicionados con la dificultad de someterse nuevamente a la evaluación de acceso a la educación superior y obligación de lograr 800 puntos, hecho que se observa es de bajas probabilidades. Las razones son claras, durante el desarrollo del curso de nivelación se trabaja con un currículo compuesto por el tronco común en donde se desarrollan las cátedras de: universidad y buen vivir, introducción a la comunicación académica; además las asignaturas generales: estudios sociales y culturales, psicología y matemáticas. Sin embargo que los estudiantes aprueban el curso, deben nuevamente presentarse a rendir la evaluación de acceso a la educación superior con las áreas de: razonamiento verbal, abstracto y numérico, éstas últimas no se trabajan en el curso de nivelación. 
También la tabla 1 muestra que únicamente hay tres carreas sin estudiantes condicionados, esto es por disposición externa a la UNACH, el SNNA considera que esas carreras no deben tener la exigencia de los 800 puntos en el examen de ingreso, pero sí el mínimo para haber alcanzado el cupo y acceder a la educación superior y cumplir el ciclo de postulación y aceptación.

Así surge la necesidad de investigar uno de los componentes del currículo oficial, la metodología; esto porque los demás componentes como: "introducción, resultados de aprendizaje, unidades de análisis, conocimientos (saber), habilidades y actitudes (saber hacer y saber ser), actividades evaluativas" (SNNA, 2014). Sí están claramente estipulados. Se considera que desde las actividades metodológicas se puede trabajar las áreas a ser evaluadas como ejes transversales y aportar a la preparación para esa rendición de la nueva evaluación.

La importancia también se resalta en que esta es la base para formular un compromiso de futuras investigaciones fundamentadas en la necesidad de hacer un seguimiento de la eficiencia de las metodologías aplicadas por los docentes con los estudiantes desde el ciclo de nivelación hasta la titulación y así determinar las fortalezas y debilidades institucionales en este elemento central del currículo.

\section{Estudio actual y trabajo previo en el área}

Como trabajo previo en el área objeto de esta investigación, se tiene información que hay un diálogo de coordinadores del ciclo de nivelación con los estudiantes para indagar sobre opiniones de los estudiantes respecto al desempeño metodológico de los docentes, eso sirve para hacer sugerencias a los docentes, pero sin la formalidad ni sistematicidad de planear, ejecutar, evaluar y tomar acciones de mejora.

No existen investigaciones previas en este campo de las metodologías de docentes del ciclo de nivelación en la UNACH, razón por la que, se genera interés por los resultados en los docentes, estudiantes y directivos.

\section{Algunos referentes teóricos relevantes}

Para el trabajo docente, "apostar por educar para la sostenibilidad significa definir un proyecto educativo de aprendizaje, organización, toma de decisiones" (Martínez, 2010). La sostenibilidad también implica el aprovechamiento de los recursos sin comprometer los necesarios y suficientes para las nuevas generaciones, hacer sostenible el trabajo docente implica interrelacionar todos los elementos y niveles del currículo y en este caso tomar como centro la metodología porque es llevar a la práctica lo planeado y conseguir los objetivos.

Hablar de enseñanza, “desde la perspectiva del pensamiento del profesor supone que enseñar no se reduce al aspecto didáctico o a lo que se realiza en clase, sino que el profesor reflexiona, analiza e interpreta aspectos intelectuales y afectivos para realizar su práctica docente" (Cañedo \& Figueroa, 2013). Eso ocurre por la naturaleza humana del docente, esto muestra la posibilidad cierta de mejorar lo que hace. 
Ya en el ámbito institucional, el Modelo Educativo, Pedagógico y Didáctico (MEPED) de la UNACH, establece la necesidad de trabajar con el paradigma de la complejidad y los enfoques sociocrítico, holístico y conectivista.

Trabajar desde el paradigma de la complejidad aflora la noción de dificultad y el consecuente rechazo aún antes de abordarlo, luego de conocerlo, se entenderá que en la realidad los fenómenos y objetos de estudio reales son complejos y que lo mejor es entender y aprovechar los fundamentos teóricos dados por el paradigma. Aquí lo conceptual entre los términos complicado y complejo, "un fenómeno complicado es aquel que es difícil de entender y de explicar. Fenómeno complejo se caracteriza por su imprevisibilidad, imposibilidad de conocer o describirlo en su totalidad, en un número finito de pasos y plazo de tiempo" (UNACH, 2014).

Con este contexto, se analiza las actividades metodológicas dadas en el currículo y de entre las utilizadas en la práctica por el docente de nivelación y se encuentra limitaciones para enmarcarse en el modelo pedagógico institucional.

En referencia a la metodología del docente, "en el ámbito universitario contar con un entorno que signifique una innovación en la metodología docente ante las demandas sociales actuales, debe ser el entorno propio del ejercicio profesional" (Piqué \& Forés, 2012). Más esto no ocurre en ese ambiente de aprendizaje asignado para los estudiantes de nivelación.

Las limitaciones institucionales no permiten cumplir a cabalidad con la dotación de verdaderos ambientes de aprendizaje.

Ante la variedad de clasificaciones de métodos y técnicas de aprendizaje, "un método de enseñanza es el conjunto de técnicas y actividades para lograr objetivos educativos, que tiene sentido como un todo y responde a una denominación conocida y compartida por la comunidad científica" (Gonzáles, 2012).

A más de lo expresado, aquí una realidad preocupante en la docencia de educación superior y no es la excepción en la UNACH, "profesionales entran en la educación superior y no tienen una formación para trabajar como profesor" (Aramasi, Nadine, \& Daniele, 2015). Cierto es que, las normativas de acceso a la docencia solo exigen título de tercer nivel o maestría para laborar en el ciclo de nivelación, pero esto lleva en algunos casos a la repitencia de prácticas docentes descontextualizadas o repetitivas con décadas pasadas que para la actualidad ya no son eficientes.

\section{Hipótesis, objetivos y enlaces a la teoría}

Cómo hipótesis central propuesta para este estudio se tiene: es significativa la relación entre la apreciación de las actividades metodológicas utilizadas por los docentes para facilitar los aprendizajes y lo referido por los estudiantes.

El objetivo del trabajo es identificar las metodologías que aplican los docentes en nivelación de la FCEHT, así como la apreciación que de esas metodologías tienen los estudiantes, con el fin de estructurar las especificaciones metodológicas para el desempeño docente. 
Esto da curso a la revisión de las teorías referentes a modelo pedagógico, currículo, metodologías, aprendizaje y enseñanza, sostenibilidad y formación docente; preceptos teóricos ya analizados.

\section{Relación de hipótesis y diseño de la investigación}

Siendo la investigación y por tanto la difusión de resultados elaborados por fases, hasta aquí está lograda la primera fase que es la recolección de la información, procesamiento, interpretación y relación con los lineamientos que da el modelo pedagógico institucional. En ese marco es un diseño no experimental, descriptivo y la hipótesis busca probar la relación entre lo realizado y expresado por los docentes con lo percibido por los estudiantes.

\section{Implicaciones teóricas y prácticas de este estudio}

Con la base en referentes de tipo estadístico, se requiere un análisis minucioso de las percepciones tanto de docentes y de estudiantes referidas a las actividades metodológicas durante el proceso de aprendizajes, esto conduce al razonamiento profundo de la sistematicidad de las metodologías, porque se presume que el docente supone que lo hecho por él fue bueno y debe logar el aprendizaje, más en la práctica el no manejar el lenguaje de las teorías científicas de procesos implica hacer una parte y no cerrar el círculo PHVA, consistente en planificar, hacer, verificar y actuar.

Trabajada esta fase se tendrá claro qué metodologías se usan y con qué intensidad y si éstas responden al modelo pedagógico. Esa afinidad o ausencia de correspondencia llevará a teorizar lo encontrado y fundamentar las soluciones.

\section{Métodos}

La investigación hasta la esta fase de difusión de los resultados y propuesta inicial es de diseño no experimental y tipo descriptivo, con el método de investigación inductivo- deductivo; la variable de estudio es las metodologías docentes.

La población fue 294 estudiantes matriculados en nivelación y muestra 80, seleccionados mediante muestreo no probabilístico de las carreras de estudiantes condicionados descritos en la tabla 1 y que pertenecen al periodo académico octubre 2016 - marzo 2017; además 25 docentes de nivelación exclusivamente de carreras de la FCEHY.

La técnica fue la encuesta, instrumento el cuestionario con 20 preguntas formuladas con las actividades metodológicas extraídas del currículo de nivelación para el área de educación. Las preguntas son para valoración cualitativa con escala Likert de opciones: siempre, casi siempre, a veces, rara vez y nunca; para el procesamiento estadístico se asigna valor numérico, así: 5, 4, 3, 2 y 1 respectivamente. Previa a la aplicación del instrumento se validó con expertos y la proporción de validez supera el $95 \%$. 
Para la aplicación de los instrumentos de investigación con cada grupo se procedió así: con estudiantes se generó una explicación sintética de la existencia de un proyecto de investigación con fines de diagnóstico y futura propuesta de mejoras de las metodologías, esto a fin de pedir contestación sincera y razonada de cada interrogante. En el caso de los docentes se aplicó un taller de trabajo para socializar íntegramente el proyecto de investigación y luego de tener el compromiso de apoyo, se aplicó el cuestionario, con la misión de socializar los resultados y proponer una metodología alternativa a la actual.

El análisis de datos fue con técnicas cuantitativas, así, la fiabilidad con el alfa de Cronbach, obteniendo para docentes 0,911 , que implica muy aceptable; mientras los datos de estudiantes arrojan 0,931 que demuestra mayor confiabilidad. Las mismas preguntas fueron aplicadas a docentes y estudiantes, con el respectivo enfoque de redacción en concordancia con la función.

Parte importante de esta investigación es el análisis de la correspondencia entre las actividades metodológicas desarrolladas y lo expresado en el modelo pedagógico, para ese fin se utilizó la lectura, análisis, síntesis y construcción de organizador gráfico.

Esta publicación es parte de la ejecución de un proyecto de investigación aprobado institucionalmente, proyecto que contiene las consideraciones éticas por investigar con seres humanos, proyecto que atravesó la revisión por pares y luego se nombró una comisión de expertos institucionales para fortalecerlo en su versión final.

\section{Resultados}

Tabla 2. Actividades metodológicas y valoración de uso por docentes y estudiantes

\begin{tabular}{rlrr}
\hline No. & \multicolumn{1}{c}{ Actividades del currículo oficial } & $\begin{array}{c}\text { Media de } \\
\text { docentes }\end{array}$ & \multicolumn{2}{c}{$\begin{array}{c}\text { Media de } \\
\text { estudiantes }\end{array}$} \\
\hline 1 & Discusiones en grupo & 4,56 & 3,34 \\
2 & Debate, discusión y reflexión & 4,76 & 3,6 \\
3 & Utilización de herramientas informáticas & 4,48 & 3,16 \\
4 & Realización de cuadros comparativos & 4,32 & 3,33 \\
5 & Realización de ensayos críticos & 4,36 & 3,23 \\
6 & Exposiciones individuales y grupales & 4,48 & 3,78 \\
7 & Desarrollo de investigaciones & 4,52 & 3,68 \\
8 & Ejecución de consultas bibliográficas & 4,44 & 3,19 \\
9 & Presentación de sociodramas & 4,24 & 2,51 \\
10 & Aplicaciones de instrumentos de investigación & 4,04 & 2,68 \\
11 & Elaboración de glosario de términos & 4,08 & 2,86 \\
12 & Propuesta creativa de solución a problemas & 4,6 & 3,33 \\
13 & Planteamiento de problemas & 4,76 & 3,71 \\
14 & Resolución de problemas propuestos & 4,68 & 3,71 \\
15 & Taller de análisis y discusión & 4,72 & 3,3 \\
16 & Presentación e interpretación de datos & 4,6 & 3,29 \\
17 & Utilización de conceptos y propiedades & 4,6 & 3,45 \\
18 & Da puntos de vista personales y considera otros & 4,72 & 3,73 \\
19 & Flexibilidad en trabajo colaborativo & 4,68 & 3,55 \\
\hline
\end{tabular}


Fuente: Cuestionario aplicados a docentes y estudiantes

Aquí la valoración de actividades metodológicas, el criterio de presentar la media es porque constituye medida representativa de los grupos participantes, por ejemplo, en "discusiones en grupo", según los docentes "casi siempre", mientras para los estudiantes es "a veces".

La figura siguiente permite observar gráficamente en un diagrama de dispersión los puntos resultantes de pares ordenados formados por las medias aritméticas de la valoración de uso de las actividades metodológicas, apreciación de los docentes y de los estudiantes.

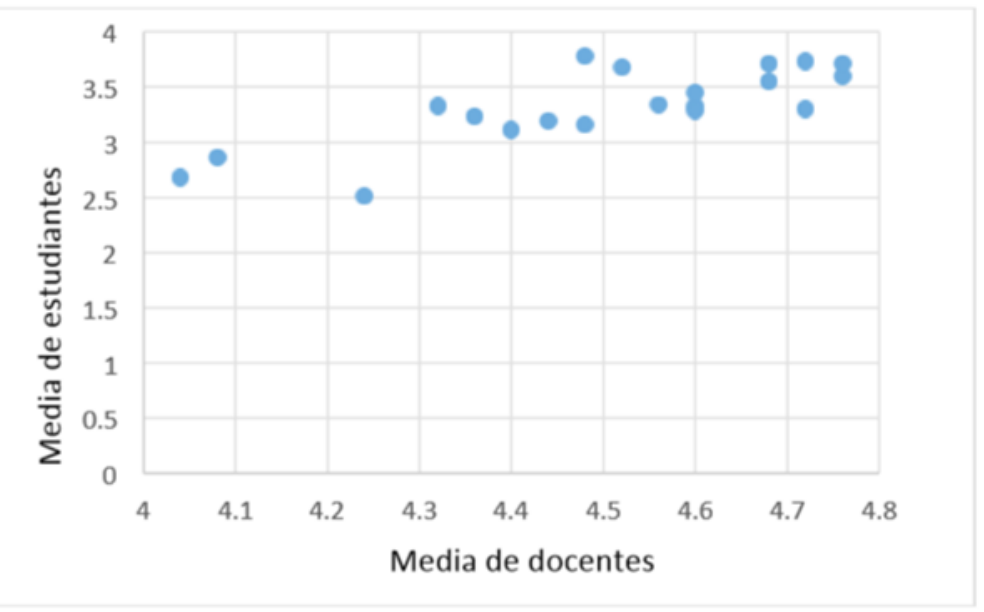

Figura 1. Gráfica de dispersión con resultados de docentes y de estudiantes

Es importante rescatar que de los datos recolectados, todas las actividades metodológicas se utilizan, además que tanto estudiantes como docentes conocen cada actividad, lo encontrado también indica que mientras los docentes creen se ejecuta con más frecuencia, para los estudiantes la frecuencia de uso es menor.

Para efectos prueba de hipótesis se utilizó el coeficiente de correlación de Pearson que es una medida de relación lineal entre variables cuantitativas, se encontró $r=0,796$, implica una correlación positiva alta entre las valoraciones. Esto prueba la hipótesis antes citada.

Se propone fortalecer las competencias pedagógicas para comprender y usar los tipos de métodos en correspondencia con las respectivas técnicas.

La función de docentes es diseñar y ejecutar actividades coherentes al currículo abierto donde se respeta la diversidad y diferencias individuales, incompatibles con el currículo rígido, común y uniforme hecho para un único tipo de estudiante. 
Otro eje son, procesos de aprendizaje: solución de problemas; trabajo colaborativo; pensamiento crítico; experimentación; formulación y prueba de hipótesis; estudios de casos; alternativas a dificultades reales.

Analizados los lineamientos para el ejercicio docente desde el modelo pedagógico institucional, se encuentra que hay variada fundamentación sociológica, antropológica, filosófica, pedagógica, didáctica, curricular y en particular al referirse a la metodología hay cuatro fases definidas; para mayor comprensión y discusión de resultados, se presenta sintéticamente en un diagrama de flujo.

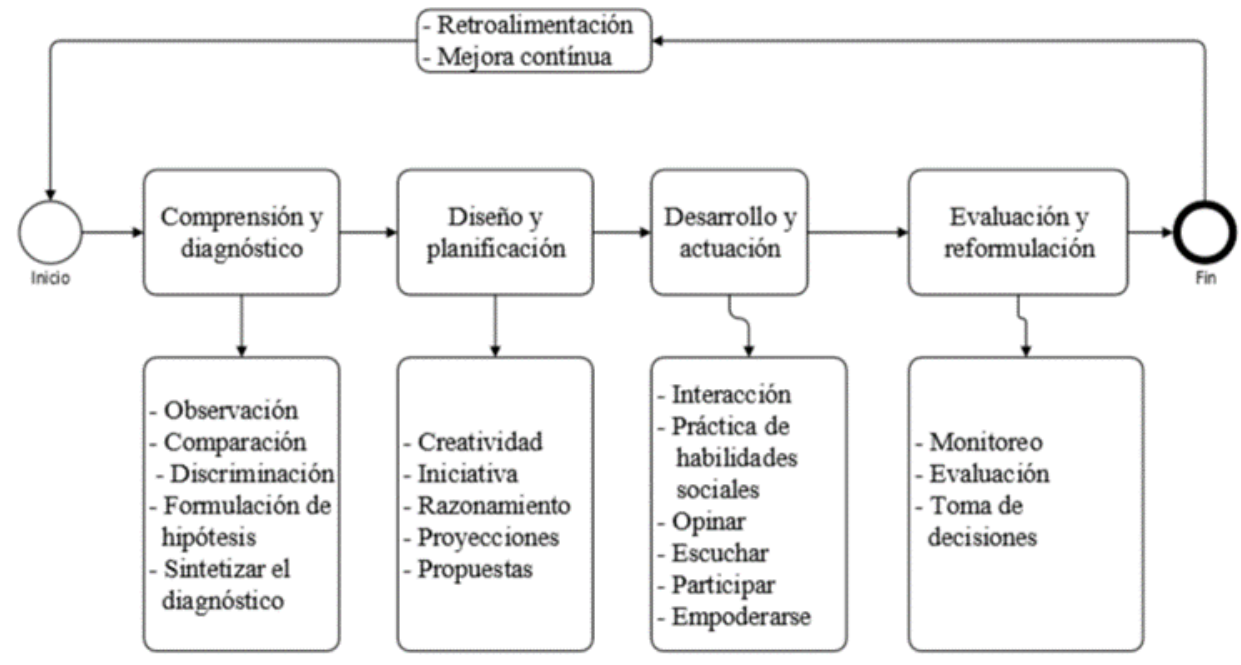

Figura 2. Diagrama de flujo con elementos del Modelo Pedagógico UNACH

\section{Discusión}

En observancia a la normativa institucional, "Los estudiantes que se matriculen en el Curso de Nivelación, no son oficialmente estudiantes de la UNACH, pero gozan de los mismos beneficios que los estudiantes regulares" (Honorable Consejo Universitario, 2013).

Se considera que gozar de los mismos derechos implica acceder a una educación concordante con la misión institucional esto es, educarse para "Crear, desarrollar, transferir y difundir el conocimiento, los saberes y la cultura” (Honorable Consejo Universitario, 2017)

Más esto en la práctica dista mucho de hacerse realidad, las actividades metodológicas que prevalecen están dadas en la tabla 2, trabajadas esporádicamente y de forma aislada, no se tornan significativas. Si se trabajarían como un sistema, tanto las fases de planificación como ejecución y evaluación serían diferentes, así se entendería que cada acción tiene una causa y efecto, que cada componente forma parte de un todo, que los procesos de aprendizajes son cíclicos; con la evaluación, no se buscaría culpables sino qué elemento del proceso falló y requiere recuperación.

\section{Conclusiones}


Hay correlación positiva alta entre la valoración de las actividades metodologías que hacen los docentes en contraste con la valoración de los estudiantes.

Las actividades metodológicas utilizadas en nivelación corresponden a una gama de actividades dispersas que desde el accionar docente están carentes de organización pedagógica.

Según los docentes, las actividades metodológicas son utilizadas "casi siempre", para los estudiantes es "a veces"; además la tabla 2 muestra que, las actividades de menor uso son, la realización de investigaciones, visitas a sitios web académicos, sociodramas, elaboración de glosarios.

Las competencias docentes para la educación deben cubrir por lo menos los campos: tecnológico, pedagógico - didáctico, social, investigativo y de liderazgo, esto permitirá fortalecer las metodologías.

\section{Agradecimientos}

Son motivo de agradecimiento los directivos de la UNACH y en particular de la FCEHT por haber asignado a los docentes en la distribución de trabajo horas dedicadas para actividades de investigación con el fin de contribuir a la solución de los problemas institucionales. De manera particular el reconocimiento por el valioso aporte como expertos para la validación de los instrumentos a: PhD Angélica Urquizo, PhD Carlos Gafas, PhD Olga Barbón. Por su singular aporte se reconoce a los estudiantes del ciclo de nivelación y sus docentes que contribuyeron respondiendo con honestidad el instrumento de investigación. Hay un mutuo agradecimiento entre los autores de esta publicación por el aporte brindado hasta este punto y el compromiso para continuar hasta aplicar una propuesta de metodologías alternativas y evaluar su validez a fin de mejorarla.

\section{Bibliografía}

Aramasi, P., Nadine, I., \& Daniele, R. (2015). Métodos de enseñanza utilizados por docentes del curso de enfermería. Enfermería Global(37). Obtenido de http://scielo.isciii.es/pdf/eg/v14n37/docencia2.pdf

Asamblea Nacional. (2010). Ley Orgánica de Educación Superior . Quito, Ecuador.

Cañedo, T., \& Figueroa, I. E. (2013). La práctica docente en educación superior: una mirada hacia su complejidad. Sinéctica(41). Obtenido de http://www.sinectica.iteso.mx/articulo/?id=41_la_practica_docente_en_educacion_su perior_una_mirada_hacia_su_complejidad

Coordinación SNNA UNACH. (2016). Oferta académica Histórica por carreras. Riobamba. Gonzáles, J. (2012). La clasificación de los métodos de enseñanza en educación superior.

Contextos $\quad$ Educativos(15), $\quad 93 \quad-\quad 106 . \quad$ Obtenido de https://publicaciones.unirioja.es/ojs/index.php/contextos/article/view/657/620 
Honorable Consejo Universitario. (2013). Reglamento de la Unidad de Nivelación y Admisión de la Universidad Nacional de Chimborazo. (U. N. Chimborazo, Ed.) Riobamba.

Honorable Consejo Universitario. (2017). Plan estratégico de desarrollo de la Universidad Nacional de Chimborazo, Riobamba. (U. N. Chimborazo, Ed.) Riobamba.

Jenaro, e. a. (2012). Metodologías docentes en la educación superior: percepciones del profesorado sobre su importancia y uso. (M. d. uso, Ed.) Obtenido de http://institucional.us.es/revistas/universitaria/39/art_2.pdf

Martínez, J. (2010). ¿Qué significa sostenibilidad para la escuela? Centro Nacional de Educación Ambienta. Universidad del País Vasco.

Piqué, B., \& Forés, A. (2012). Propuestas metodológicas para la educación superior.

Barcelona, España : Universitat de Barcelona. Obtenido de http://hdl.handle.net/2445/30702

SNNA. (2014). Microcurrículos por áreas de conocimiento. Quito, Ecuador.

SNNA. (01 de 12 de 2017). Sistema Nacional de Nivelación y Admisión. Obtenido de http://www.snna.gob.ec/wp-content/themes/institucion/snna_menu.php

UNACH. (2014). Modelo Educativo, Pedagógico y Didáctico (Vol. 1). Riobamba, Chimborazo, Ecuador. 